\title{
Soft QCD Results from CDF
}

\author{
Christina Mesropian* ${ }^{* \dagger}$ \\ Rockefeller University \\ E-mail: christina.mesropian@cern.ch
}

\begin{abstract}
We review the latest underlying event and minimum bias results and discuss the associated problematics with the idea of establishing a solid baseline for the LHC experiments. These measurements include study of the underlying event in Drell-Yan and dijet events, and inclusive differential (in $p_{T}$ ) cross sections of centrally $(|\eta|<1)$ produced lambdas, cascades and omegas. We also present recent results on diffraction obtained by the CDF collaboration. Single-diffractive W and $\mathrm{Z}$ production is discussed. The first experimental observation of exclusive dijets, exclusive $\chi_{c 0}$ mesons, and a search for exclusive diphotons are discussed. We also present results from a study of central rapidity gap production in soft and hard diffractive events.
\end{abstract}

35th International Conference of High Energy Physics

July 22-28, 2010

Paris, France

* Speaker.

${ }^{\dagger}$ for the CDF Collaboration 


\section{Underlying Event and MinBias Studies}

The existence of Monte Carlo models that simulate accurately QCD hard-scattering events is essential for all new physics searches at hadron-hadron colliders. To achieve a given accuracy one should be able not only to have a good model of the hard scattering part of the process, but also of the beam-beam remnants (BBR) and the multiple parton interactions (MPI), an unavoidable background to most collider observables. For Drell-Yan production, the final state includes a lepton-antilepton pair, and there is no colored final state radiation, thus providing a clean way to study the underlying event (UE). The methodology of the presented study is similar to previous CDF UE studies [1], by considering toward, away, and transverse regions defined by the azimuthal angle $\Delta \phi$ relative to the direction of the leading jet in the event, or the direction of the lepton-pair in Drell-Yan production $\left(\Delta \phi=\phi-\phi_{j e t_{1} / \text { pair }}\right)$. We study charged particles with $p_{T}>0.5 \mathrm{GeV} / \mathrm{c}$ and $|\eta|<1$ in the above-mentioned regions. For high- $p_{T}$ jet production we require that the leading jet in the event, reconstructed with the MidPoint algorithm, have $\left|\eta_{\text {jet }}\right|<2$. For Drell-Yan production we require the invariant mass of the lepton-pair to be in the mass region of the Z-boson, $70<M_{\text {pair }}<110 \mathrm{GeV} / \mathrm{c}^{2}$, with $\left|\eta_{\text {pair }}\right|<6$. The underlying event observables are found to be reasonably flat with the increasing lepton pair transverse momentum in the transverse and toward regions, but distributions go up in the away region to balance lepton pairs. In Fig. 1(a) and (b), we plot two

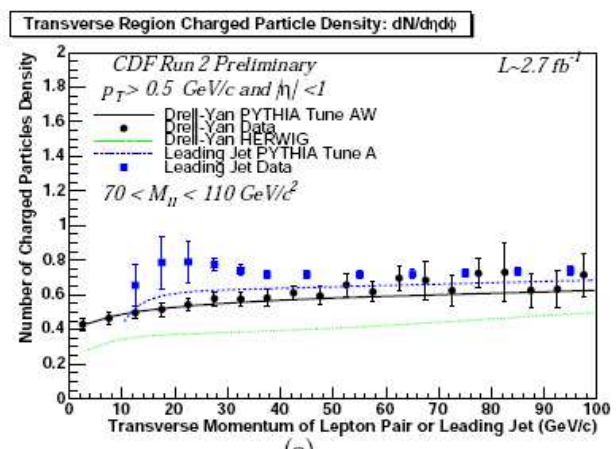

(a)

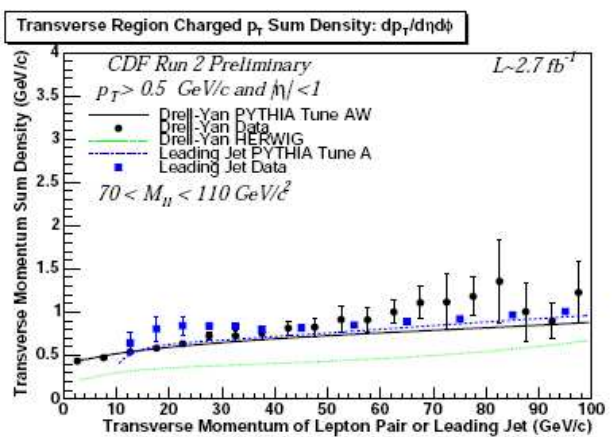

(b)

Figure 1: The underlying event observables in Drell-Yan production.

observables corresponding to the underlying event: the charged particle number density and the charged transverse momentum sum density in the transverse region, compared with PYTHIA Tune A and AW [2]-[3], HERWIG [4] without MPI and a previous results of CDF analysis of underlying events with the leading jet. There is very good agreement with PYTHIA tune AW MC predictions, while HERWIG produces much less activity. The comparison with leading jet underlying event results shows close agreement, which indicates the universality of underlying event modeling. The behavior of the average charged-particle $p_{T}$ versus charged-particle multiplicity is also important. The rate of change of $p_{T}$ versus charged multiplicity is a measure of the amount of hard versus soft processes contributing, and it is sensitive to the modeling of the multiple-parton interactions. PYTHIA Tune A and Tune AW do a good job in describing the data on $\left\langle p_{T}\right\rangle$ versus multiplicity for minbias and Drell-Yan events. The behavior of $\left\langle p_{T}\right\rangle$ versus multiplicity is remarkably similar for minbias events and Drell-Yan events, suggesting that MPI are playing an important role in both these processes. Models with MPI predict that the underlying event will become much more 
active at the LHC. A lot can be learned by comparing the Tevatron results with early measurements at the LHC, to improve modeling of the underlying event for future precision measurements.

\subsection{Hyperons}

We also report a set of measurements of inclusive invariant $p_{T}$ differential cross sections of $\Lambda^{0}, \bar{\Lambda}^{0}, \Xi^{ \pm}$, and $\Omega^{ \pm}$hyperons reconstructed in the central region with $|\eta|<1$ and $p_{T}<10 \mathrm{GeV} / \mathrm{c}$. Events were collected with a minimum-bias trigger. As $p_{T}$ increases, the slopes of the differential cross sections remain similar, which could indicate universality of particle production in $p_{T}$. The production ratios $\Xi^{-} / \Lambda^{0}$ and $\Omega^{-} / \Lambda^{0}$ were also studied as a function of $p_{T}$ and are fairly constant in the high $p_{T}$ region.

\section{Diffractive results}

The CDF collaboration contributed extensively [5]-[13] to significant progress in understanding diffraction by studying a wide variety of diffractive processes at three different center-of-mass energies: $630 \mathrm{GeV}, 1800 \mathrm{GeV}$, Run I of Tevatron, and $1960 \mathrm{GeV}$ - Run II. Some important results include the observation of QCD factorization breakdown in hard single diffractive processes, discovery of large rapidity gaps between two jets, study of diffractive structure function in double pomeron exchange dijet events. Diffractive W/Z production is an important process for probing the quark content of the pomeron, since to leading order, the W/Z is produced through a quark, while gluon associated production is suppressed by a factor of $\alpha_{S}$ and can be identified by an additional jet. CDF studied diffractive $\mathrm{W}$ production in Run I [6] by using the rapidity gap signature of diffractive events. In Run II, we select events with "intact leading antiproton" signature, where $\bar{p}$ is detected in the Roman Pot Spectrometers (RPS). The RPS allows very precise measurement of the fractional momentum loss of $\bar{p}(\xi)$, eliminating the problem of gap survival probability. The novel feature of the analysis, the determination of the full kinematics of the $W \rightarrow l v$ decay, is made possible by obtaining the neutrino $E_{T}{ }^{v}$ from the missing $E_{T}, E_{T}$, and $\eta_{v}$ from the formula $\xi^{R P S}-\xi^{c a l}=\frac{E_{T}}{\sqrt{s}} e^{-\eta_{v}}$, where $\xi^{R P S}$ is the true $\xi$ measured in RPS and $\xi^{\text {cal }}=\sum_{i(\text { towers })}\left(E_{T}^{i} / \sqrt{s}\right) \exp \left(-\eta^{i}\right)$. The fractions of diffractive $\mathrm{W}$ and $\mathrm{Z}$ events are measured to be $[0.97 \pm 0.05$ (stat. $) \pm 0.11$ (syst.) $] \%$ and $[0.85 \pm 0.20$ (stat. $) \pm 0.11$ (syst. $)] \%$ for the kinematic range $0.03<\xi<0.10$ and $|t|<1 \mathrm{GeV} / \mathrm{c}$. The measured diffractive $\mathrm{W}$ fraction is consistent with the Run I CDF result when corrected for the $\xi$ and $t$ range.

\subsection{Central Exclusive production}

The exclusive dijet production was first studied by CDF in Run I data and a limit of $\sigma_{\text {excl }}<3.7$ $\mathrm{nb}(95 \% \mathrm{CL})$ was placed [14]. This study was continued in Run II when the observation of the exclusive dijet production was reported [15]. The exclusive signal is extracted using the dijet mass fraction method: the ratio $R_{j j} \equiv M_{j j} / M_{X}$ of the dijet mass $M_{j j}$ to the total mass $M_{X}$ of the final state is formed and used to discriminate between the signal of exclusive dijets, defined as $R_{j j}>0.8$, and the background of inclusive double pomeron exchange dijets, expected to have a continuous distribution concentrated at lower $R_{j j}$ values. The measured cross sections [15] are consistent with predictions by Khoze et al. [16]. 
Another process which is closely related to exclusive Higgs production is exclusive diphoton production $p \bar{p} \rightarrow p \gamma \gamma \bar{p}$. CDF has performed a search for exclusive $\gamma \gamma$ using a $0.5 \mathrm{fb}^{-1}$ data sample obtained with a trigger requiring the presence of two electromagnetic (EM) towers and forward gaps in both forward directions. Three candidate events were found, by requiring all calorimeters to be empty, and no tracks to be associated with two EM trigger towers. Two of these events are likely to be $\gamma \gamma$, and the third is more likely to be $\pi^{0} \pi^{0}$. A limit was placed on exclusive diphoton production of $410 \mathrm{fb}$ at $95 \%$ CL [17]. The prediction [18] is compatible with this limit. CDF plans to update this measurement with additional available data.

CDF II also studied dimuon production, when the event signature requires two oppositely charged central muons, and either no other particles (large forward rapidity gaps), or one additional photon detected. Within the kinematic region $|\eta(\mu)|<0.6$ and $M_{\mu \mu} \in[3.0,4.0] \mathrm{GeV} / c^{2}$, there are 402 events with no EM shower, see the $M_{\mu \mu}$ spectrum in Fig. 2(left).

The $J / \psi$ and $\psi(2 S)$ are prominent, together with a continuum. By requiring one EM shower with $E_{T}^{E M}>80 \mathrm{MeV}$ in addition to the requirement mentioned above, we are able to measure $\chi_{c 0}$ production. Allowing EM tower causes a large increase (+ 66 events) in the Jpsi peak and minor change ( +1 event) in the $\psi(2 S)$ peak. After correcting for background, efficiencies, and the branching fraction, we obtain a cross section for exclusive $\chi_{c 0}$ production of $75 \pm 10$ (stat) \pm 10 (syst) $\mathrm{nb}$ [19], which is compatible with the theoretical predictions [20]-[21].

\subsection{Double Diffractive processes}

Double diffractive (DD) dissociation is the process in which two colliding hadrons dissociate into clusters of particles (jets in case of hard DD dissociation) producing events with a large nonexponentially suppressed central pseudo-rapidity gap.
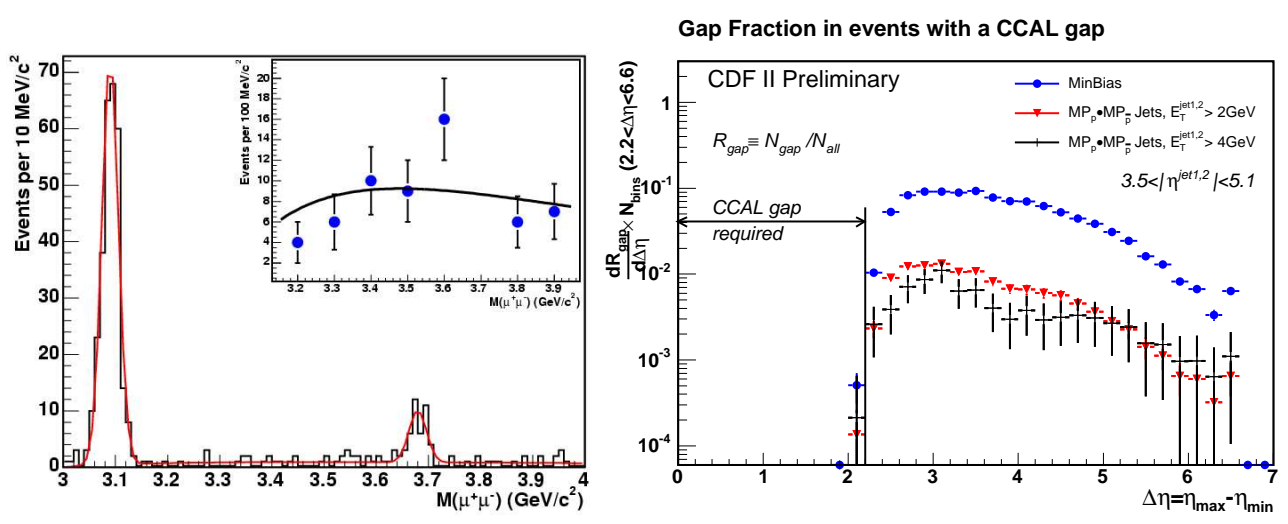

Figure 2: (left) Mass $M_{\mu \mu}$ distribution of 402 exclusive events, with no EM shower, (histogram) together with a fit to two Gaussians for the $J / \psi$ and $\psi(2 S)$, and a QED continuum. All three shapes are predetermined, with only the normalizations floating. Inset: Data above the $J / \psi$ and excluding $3.65<M_{\mu \mu}<3.75$ $\mathrm{GeV} / \mathrm{c}^{2}(\psi(2 S))$ with the fit to the QED spectrum times acceptance (statistical uncertainties only); (right) The distribution of the gap fraction $R_{\text {gap }}=N_{\text {gap }} / N_{\text {all }}$ vs. $\Delta \eta=\eta_{\max }-\eta_{\min }$ for min-bias and MiniPlug jet events of $E_{T}^{\text {jet } 1,2}>2 \mathrm{GeV}$ and $E_{T}^{\text {jet } 1,2}>4 \mathrm{GeV}$.

The extended rapidity coverage provided by the MiniPlug calorimeters $(3.5<|\eta|<5.1)$ makes CDF II a powerful detector for DD studies. The data sample where jets are required in MP 
calorimeters is used to study "hard" diffraction production. "Soft" diffractive production is analyzed by examining low luminosity data collected with a min-bias trigger. Fig. 2(right) shows a comparison of the gap fraction, as a function of $\Delta \eta$, between "hard" and "soft" DD production when CCAL gap, a rapidity gap within $-1.1<\eta<1.1$, is required. This comparison is relatively free of systematic uncertainties, as detector and beam related effects cancel out. The distributions are similar in shape, demonstrating that the gap fraction decreases with increasing $\Delta \eta$ for both "hard" and "soft" DD productions.

\section{References}

[1] T. Affolder et al.,Phys. Rev. D65 092002 (2002)

[2] T. Sjöstrand, Phys.Lett B157 321 (1985); M. Bengtsson, T. Sjöstrand, and M. van Zijl, Z. Phys. C32 67 (1987); T. Sjöstrand et al., Computer. Physics Commun. 135, 238 (2001).

[3] R. Field and R.C. Group (2005) hep-ph0510198; R. Field, Proceedings of TeV4LHC 2006 Workshop 4th meeting, Batavia, Illinois, 20-22 Oct.2006.

[4] G. Marchesini and B.R. Webber, Nucl.Phys B310, 461 (1988); S. Catani, G. Marchesini, and B.R. Webber, Nucl.Phys B349, 635 (1991).

[5] F. Abe et al., Phys.Rev.Lett. 74, 855 (1995).

[6] F. Abe et al., Phys.Rev.Lett. 78, 2698 (1997).

[7] F. Abe et al., Phys.Rev.Lett. 79, 2636 (1997).

[8] F. Abe et al., Phys.Rev.Lett. 80, 1156 (1998).

[9] F. Abe et al., Phys.Rev.Lett. 81, 5278 (1998).

[10] T. Affolder et al., Phys.Rev.Lett. 84, 5043 (2000).

[11] T. Affolder et al., Phys.Rev.Lett. 85, 4215 (2000).

[12] T. Affolder et al., Phys.Rev.Lett. 87, 141802-1 (2001).

[13] T. Affolder et al., Phys.Rev.Lett. 87, 241802-1 (2001).

[14] T. Affolder et al., Phys.Rev.Lett. 85, 4215 (2000).

[15] T. Aaltonen et al., Phys.Rev. D 77052004 (2008).

[16] V.A. Khoze et al., Eur. Phys.J. C 14, 525 (2000); V.A. Khoze et al., arXiv:0705.2314.

[17] T. Aaltonen et al., Phys. Rev. Lett. 99242002 (2007).

[18] V.A. Khoze et al., Eur. Phys. J. C 38, 475 (2005).

[19] T. Aaltonen et al., Phys.Rev.Lett. 102242001 (2009).

[20] V.A. Khoze et al., Eur.Phys.J. C 35211 (2004); V.A. Khoze et al., Eur.Phys.J. C 19 599(E) (2001).

[21] A. Bzdak, Phys.Lett. B 619, 288 (2005). 